\title{
Assessment of National Poison Data System Algorithms to identify Public Health Events
}

\author{
Royal K. Law*1, Howard Burkom², Alvin Bronstein ${ }^{3}$ and Josh Schier ${ }^{1}$ \\ ${ }^{1}$ Centers for Disease Control and Prevention, Chamblee, GA, USA; 2 Johns Hopkins Applied Physics Laboratory, Laurel, MD, USA; \\ ${ }^{3}$ American Association of Poison Control Centers, Alexandria, VA, USA
}

\section{Objective}

To compare the effectiveness of current surveillance algorithms used in the National Poison Data System (NPDS) to identify incidents of potential public health significance with 1) new algorithms using expanded NPDS surveillance capabilities and 2) methods beyond the NPDS' generalized historical limits model.

\section{Introduction}

NPDS is a near real-time surveillance system and national database operated by the American Association of Poison Control Centers. NPDS receives records of all calls made to the 55 regional US poison centers (PCs). The Centers for Disease Control and Prevention (CDC) use NPDS to 1) provide public health surveillance for chemical, radiological and biological exposures and illnesses, 2) identify early markers of chemical, radiological, and biological incidents, and 3) find potential cases and enhance situational awareness during a known incident. Anomalies are reviewed daily by a distributed team of PC medical and clinical toxicologists for potential incidents of public health significance (IPHS). Information on anomalies elevated to IPHS is promptly relayed to state epidemiologists or other designated officials for situational awareness and public health response.

Current NPDS surveillance algorithms utilize the Historical Limits Method, which identifies a data anomaly when call volumes exceed a statistical threshold derived from multiple years of historical data [ref]. Alternative analysis tools such as those employed by ESSENCE and other computerized data surveillance systems have been sought to enhance NPDS signal analysis capability. Technical improvements have been implemented in 2013 to expand NPDS surveillance capabilities but have not been thoroughly tested. Moreover, other data aberration detection algorithms, such as temporal scan statistics, have not yet been tested on real-time poison center data.

\section{Methods}

The data series for this assessment are 7-year historical time series of NPDS hourly call counts. NPDS catalogues confirmed PHS call clusters each year for current surveillance algorithms. New algorithm strategies will be run on the same data series to compare with current algorithm strategies. These new strategies include the use of the newly expanded NPDS surveillance capabilities and new methods (e.g. scan statistics) beyond NPDS' historical limits model. The same methodology will be used for substance-specific surveillance, including carbon monoxide (CO) exposures and food poisonings.

To focus on missed cluster-type concerns, scenario-driven call clusters will be injected into the data series. PHS identification sensitivity and non-PHS alert rates will be balanced to assess the ability to capture historical and injected call clusters of desired exposure types for each type of surveillance algorithm. For example, performance of an algorithm with higher sensitivity and lower nonPHS alert rates will be considered superior.

\section{Results}

The collected data series includes hourly time series of general poison center call volumes and substance-specific subseries, such as $\mathrm{CO}$ exposure and food poisoning. Selected inject scenarios are under discussion, including a latency model to capture the problem of delayed uploads. R code implementing the generalized algorithm queries is written and will be shared. Summaries of NPDS-specific evaluation measures, such as positive predictive value, defined as counts of PHS clusters divided by all clusters, will be presented for method comparison using both historical IPHS and injected call clusters.

\section{Conclusions}

Based on the results, the authors will determine the most effective algorithms for identifying IPHS without overburdening the NPDS surveillance team with high quantities of anomalies. This work will improve surveillance activities using NPDS and may be helpful for health departments that conduct surveillance using poison center data.

\section{Keywords}

toxicosurveillance; poison control center; NPDS

\section{References}

Stroup, D.F., et al., Evaluation of a method for detecting aberrations in public health surveillance data. Am J Epidemiol, 1993. 137(3): p. $373-80$
*Royal K. Law
E-mail: hua1@cdc.gov 